\title{
FREE FINITE GROUP ACTIONS ON $S^{3}$
}

\author{
BY RONNIE LEE ${ }^{1}$ AND CHARLES THOMAS \\ Communicated by Glen E. Bredon, August 2, 1972
}

In this paper we describe the first stages of a theory of 3-manifolds with finite fundamental group. The strong conjecture that any free finite group action on $S^{3}$ is conjugate to a linear action is known for some cyclic groups, see [3], [4], and is supported by recent work of one of us on fundamental groups [2]. Here we concern ourselves with the weaker conjecture that any compact 3-manifold with finite fundamental group is homotopy equivalent to a Clifford-Klein form. (Note that both conjectures are phrased to avoid problems with homotopy 3-spheres.) It is known, see for example [6], that the homotopy type of such a manifold is determined by the fundamental group and the first $k$-invariant. By exploiting the link between $k$-invariant and finiteness obstruction we are able to decide which homotopy types correspond to finite Poincaré complexes, and thus restrict the possible homotopy types for manifolds. There are nonstandard types for some groups, and a corollary of our argument is the existence in dimensions $4 n-1, n \geqq 2$, of free actions homotopically distinct from orthogonal ones. When $n=1$, we can only produce such an action on a homology sphere, and it would be most interesting to know the fundamental group.

1. Homotopy type of space forms. Let the abstract group $\pi$ be isomorphic to the fundamental group of a compact 3-dimensional manifold of constant positive curvature (Clifford-Klein form), and suppose $\pi$ cannot be decomposed as a direct product. The possibilities for $\pi$ are listed in the following table, see [10, Chapter 7, p. 224]:

If $Y$ is a 3-dimensional $\mathrm{CW}$-complex such that $\tilde{Y}$ is homotopy equivalent to $S^{3}$, and we can choose an isomorphism $\psi: \pi_{1}(Y, y) \rightarrow \pi$, we shall call $Y$ a Poincaré space form. $Y$ is not necessarily finite, and the isomorphism $\psi$, although not natural, is assumed fixed. Homotopy classes of space forms are in (1-1) correspondence with the orbits in $H^{4}(\pi, \boldsymbol{Z})$ under the action of \pm Aut $\pi[6$, Theorem 1.8] and there is a well-defined obstruction to finding a finite complex in a given homotopy type, lying in the projective class group $\widetilde{K}_{0}(Z \pi)[\mathbf{8}$, Theorem $\mathrm{F}]$. We can describe this obstruc-

AMS (MOS) subject classifications (1970). Primary 57A10, 57E25; Secondary 55G45, 16A $54,55 \mathrm{~A} 25$.

Key words and phrases. 3-dimensional Clifford-Klein form, $k$-invariant, finiteness obstruction, Mayer-Vietoris sequence in algebraic $K$-theory, surgery obstruction.

${ }^{1}$ The first author was partially supported by NSF grant No. 9452 and NSF-GP-25737X. 


\begin{tabular}{cclc}
\hline$\pi$ & Generators & \multicolumn{1}{c}{ Relations } & Order \\
\hline$Z_{m}$ & $A$ & $A^{m}=1$ & $m$ \\
\hline$Q_{4 n}$ & $A, B$ & $A^{2 n}=1, A^{n}=B^{2}, B^{-1} A B=A^{-1}$ & $4 n$ \\
\hline$T_{v}^{*}$ & $A, P, Q$ & $A^{3^{v}}=1, P^{4}=1, P^{2}=Q^{2}$, & $8.3^{v}$, \\
& & $P Q P^{-1}=Q^{-1}, A P A^{-1}=Q, A Q A^{-1}=P Q$ & $v \geqq 1$ \\
\hline $0^{*}$ & $A, P, Q, R$ & As for $T_{1}^{*}$, also $R^{2}=P^{2}$, & 48 \\
\hline$I^{*}$ & $A, B, C$ & $R P R^{-1}=Q P, R Q R^{-1}=Q^{-1}, R A R^{-1}=A^{-1}$ & \\
\hline
\end{tabular}

TABLE 1

tion explicitly in the following way. Since $\pi$ is isomorphic to the fundamental group of some space form, the chains of the universal cover define an exact sequence of finitely generated $\pi$-modules

$$
0 \rightarrow Z \rightarrow F_{3} \rightarrow F_{2} \rightarrow F_{1} \rightarrow F_{0} \rightarrow Z \rightarrow 0,
$$

whose chain homotopy type corresponds to a generator $k_{0}$ of $H^{4}(\pi, Z)$. If $r$ is any integer prime to $[\pi: 1]$, and $\Sigma=\sum_{g \in \pi} g$, let $[r, \Sigma]$ be the projective ideal of $Z \pi$ generated by $r$ and $\Sigma$. There is an isomorphism, see [5, Lemma 6.1],

given by

$$
\boldsymbol{Z}+\boldsymbol{Z}[\pi] \stackrel{\sim}{\rightrightarrows} \boldsymbol{Z}+[r, \Sigma]
$$

$$
(1,0) \mapsto(\bar{r}, m \Sigma) \quad \& \quad(0,1) \mapsto(1, r), \quad r \bar{r}=1+m[\pi: 1] .
$$

It is easy to construct a map of degree $\bar{r}(\bmod [\pi: 1])$ between the chain complex above and the modified complex

$$
0 \rightarrow Z \rightarrow F_{3}+Z \pi \rightarrow F_{2}+[r, \Sigma] \rightarrow F_{1} \rightarrow F_{0} \rightarrow Z \rightarrow 0,
$$

a geometric realisation of which corresponds to the generator $\bar{r} k_{0}$ of $H^{4}(\pi, Z)$. Since $[\bar{r}, \Sigma]+[r, \Sigma]$ is free, this shows that $[\bar{r}, \Sigma]$ is the finiteness obstruction for this homotopy type.

Divide the homotopy classes of Poincaré space forms $Y$ (equivalently orbits in $H^{4}(\pi, Z)$ ) into three types:

I, $Y$ is infinite, hence not equivalent to a manifold,

$\mathrm{F}, Y$ is finite, and

$\mathrm{CK}, Y$ is equivalent to one of the classical Clifford-Klein forms.

The argument above shows that we may distinguish between types I and $\mathrm{F}$ by means of the modules $[r, \Sigma]$; the orbit of $r k_{0}$ belongs to $\mathrm{F}$ if and 
only if $[r, \Sigma] \sim 0$ in $\widetilde{K}_{0}(Z \pi)$.

THEOREM 1. If the 2-Sylow subgroups of $\pi$ are cyclic, $[r, \Sigma] \sim 0$ for all $r$. If the 2-Sylow subgroups of $\pi$ are generalised quaternion, $[r, \Sigma] \sim 0$ provided $r \equiv \pm 1(8)$.

Proof. We outline the argument for cyclic and quaternion groups, since these are typical. Observe that the module $[r, \Sigma] \sim 0$ if there is an element in the orbit of $r k_{0}$ coming from a form defined by a fixed point free representation of $\pi$. For convenience we identify $H^{4}(\pi, Z)$ with $Z_{[\pi: 1]}$ by mapping the generator $k_{0}$ to 1 . The symbol $\varphi$ denotes Euler's function, and $U$ the group of units.

$Z_{m}:[r, \Sigma] \sim 0$, since the homotopy type contains the lens space $L(m, \bar{r})$.

$Q_{4 n}$ : (i) $n=2$. If $H=Z[i, j, k] \subseteq U\left(S^{3}\right)$, one looks at the exact sequence

$$
\rightarrow K_{1}(H)+U\left(Z\left(2 Z_{2}\right)\right) \rightarrow U\left(F_{2}\left(2 Z_{2}\right)\right) \rightarrow \tilde{K}_{0}(\Lambda) \rightarrow \text {, }
$$

and sees that $[3, \Sigma]$ comes from a unit in $F_{2}\left(2 Z_{2}\right)$, which is not hit by any element in $K_{1}(H)$. This computation was originally performed by one of us and C. T. C. Wall.

(ii) $n=2 t$, and $Q_{4 n} \supseteq Q_{8}$. If $r \equiv \pm 3(8)$ the orbit of $r$ lifts to the orbit of 3 in $Z_{8}$, which shows that the covering complex with fundamental group $Q_{8}$ cannot be finite. The remaining modules are the trivial finiteness obstructions associated with the CK-forms. Each of these is defined by a fixed point free representation corresponding to minus a square in $U\left(Z_{8 t}\right)$.

(iii) $n=2 t+1$. As in (i) we decompose $\Lambda$ to show $[r, \Sigma] \sim 0$ always; hence for these groups, there are "bad" finite complexes.

We present the geometric implications of our theorem in Table 2 below. Note that the figures refer to numbers of generators rather than to number of orbits under the \pm Aut $\pi$ action.

2. Homotopically exotic actions. The argument of the previous paragraph implies the existence of finite Poincaré complexes homotopically distinct from manifolds of constant positive curvature in all dimensions $\equiv 3$ (4). Furthermore the Spivak normal fibration admits a PL-bundle structure in all cases, see [7], and the problem of finding a PL-manifold in the homotopy type reduces to one of surgery. The following theorem solves this problem for certain metacyclic groups and shows the existence of homotopically exotic, free, piecewise linear actions on spheres.

THEOREM 2. Let $n$ be an odd prime and $Q_{4 n}$ the generalised quaternion group of order $4 n$. Then, for every integer $l>0$, there exists a PL, closed, oriented $(4 l+3)$-manifold $M$ with the following properties:

(i) $\pi_{1} M=Q_{4 n}$, 


\begin{tabular}{cccccc}
\hline$\pi$ & $\varphi[\pi: 1]$ & I & CK & F but not CK \\
\hline $\boldsymbol{Z}_{m}$ & $\varphi(m)$ & - & $\varphi(m)$ & - \\
\hline$n=2^{k}(2 t+1)$ & $2^{k-1} \varphi(2 t+1)$ & $2^{k-2} \varphi(2 t+1)$ & $2^{k-2} \varphi(2 t+1)$ & - \\
$Q_{4 n}$ & $k \geqq 1$ & & & & \\
\cline { 2 - 6 } & $n \equiv 1(2)$ & $2 \varphi(n)$ & - & $\varphi(n)$ & $\varphi(n)$ \\
\hline & $T_{1}^{*}, 0^{*}, I^{*}$ & $8,16,32$ & $4,8,16$ & $2,4,4$ & \\
\hline$T_{*}^{*}, v \geqq 2$ & $8.3^{v-1}$ & $4.3^{v-1}$ & $2.3^{v-1}$ & \\
\hline
\end{tabular}

TABLE 2

(ii) the universal cover $\tilde{M}$ is $S^{4 l+3}$, and

(iii) $\pm k$-invariant of $M$ is not a square modulo $4 n$.

If $l=0$, there exists a free action of $Q_{4 n}$ on a homology 3-sphere, such that the $k$-invariant of the action satisfies (iii).

Proof. Let $D_{2 n}=\left\{A, B: A^{n}=B^{2}=1, B A B^{-1}=A^{-1}\right\}$ be the dihedral group of order $2 n$, and let $Z\left[e^{2 \pi i / n}, j\right]$ be the subring of the quaternion numbers generated by $e^{2 \pi i / n}$ and $j$. There is a cube of ring homomorphisms (shown in collapsed form),

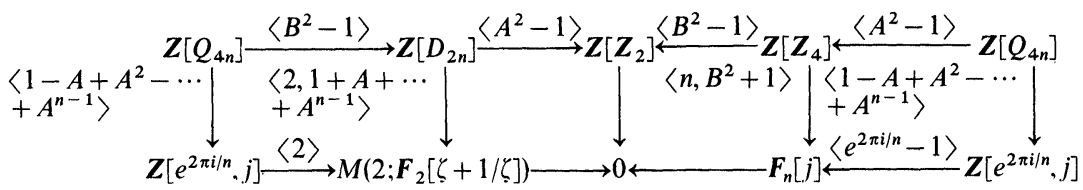

which leads to an exact sequence of Wall groups:

$$
\begin{aligned}
0 & \rightarrow L_{3}\left(\boldsymbol{Z}\left[Q_{4 n}\right]\right) \stackrel{\mathscr{x}}{\rightarrow} L_{3}\left(\boldsymbol{Z}\left[\boldsymbol{Z}_{4}\right]\right) \oplus L_{3}\left(\boldsymbol{Z}\left[\boldsymbol{D}_{2 n}\right]\right) \oplus L_{3}\left(\boldsymbol{Z}\left[e^{2 \pi i / n}, j\right]\right) \\
& \rightarrow L_{\mathbf{3}}\left(\boldsymbol{F}_{n}[j]\right) \oplus L_{\mathbf{3}}\left(\boldsymbol{Z}\left[\boldsymbol{Z}_{2}\right]\right) \oplus L_{3}\left(M\left(2 ; \boldsymbol{F}_{2}[\zeta+1 / \zeta]\right)\right) \rightarrow \cdots .
\end{aligned}
$$

We look at the image of our surgery obstruction under $\mathscr{X}$. According to H. Bass, I. Berstein and others, the Wall group of $Z_{4}$ is cyclic of order 2, and by the calculation in $[\mathbf{1}]$, an element in $L_{3}\left(Z\left[D_{2 n}\right]\right)$ is detected by its image in $L_{3}\left(\boldsymbol{Z}\left[\boldsymbol{Z}_{2}\right]\right)$ and semicharacteristic classes. The third group $L_{3}\left(Z\left[e^{2 \pi i / n}, j\right]\right)$ can be studied through the homomorphism Spin: $L_{3}\left(Z\left[e^{2 \pi i / n}, j\right]\right) \rightarrow \boldsymbol{Q}[2 \cos (2 \pi / n)]^{x} / \begin{aligned} & \text { modulo subgroup generated } \\ & \text { by units and squares in } \\ & \boldsymbol{Z}[2 \cos (2 \pi / n)]\end{aligned}$

defined by the spinor norm, see [9]. Spin is actually injective, and for the problem on hand, one can show that both the spinor norm and the semi- 
characteristic classes are zero. Possibly by changing the normal invariant we can kill off any obstruction in $L_{3}\left(Z\left[Z_{4}\right]\right)$, and by exactness the theorem follows.

\section{REFERENCES}

1. R. Lee, The spherical space form problem, Yale University Notes, New Haven, Conn., 1970

2. R. Lee, Semicharacteristic classes, Topology (to appear).

3. G. R. Livesay, Fixed point free involutions on the 3-sphere, Ann. of Math. (2) 72 (1960), 603-611. MR 22 \# 7131.

4. P. M. Rice, Free actions of $Z_{4}$ on $S^{3}$, Duke Math. J. 36 (1969), 749-751. MR 40 \# 2064.

5. R. G. Swan, Periodic resolutions for finite groups, Ann. of Math. (2) 72 (1960), 267-291. MR 23 \# A2205.

6. C. B. Thomas, The oriented homotopy type of compact 3-manifolds, Proc. London Math. Soc. (3) 19 (1969), 31-44. MR 40 \#2088.

7. C. B. Thomas and C. T. C. Wall, The topological spherical space form problem. I, Compositio Math. 23 (1971), 101-114.

8. C. T. C. Wall, Finiteness conditions for CW-complexes, Ann. of Math. (2) 81 (1965), 56-69. MR 30 \# 1515.

9. G. E. Wall, The structure of a unitary factor group, Inst. Hautes Études Sci. Publ. Math. No. 1 (1959), 59 pp. MR 21 \#3517.

10. J. A. Wolf, Spaces of constant curvature, McCraw Hill, New York, 1967. MR 36 \#829.

Department of Mathematics, Yale University, New Haven, Connecticut 06520

Current address (Ronnie Lee): University College London, Gower Street, WC1E 6BT, England. 